\title{
CYSTIC OPTIC NERVE MENINGIOMA PRESENTING WITH ACUTE PROPTOSIS
}

\author{
ROGER D. LAITT ${ }^{1}$, BIMAL KUMAR ${ }^{2}$, BRIAN LEATHERBARROW ${ }^{2}$, \\ RICHARD E. BONSHEK ${ }^{3}$ and ALAN JACKSON ${ }^{4}$ \\ Manchester
}

\begin{abstract}
SUMMARY
A 37-year-old woman presented with acute left-sided proptosis. Magnetic resonance imaging demonstrated a solid intraconal mass lesion with an associated 'capping' cyst. This lesion was resected and found on histological examination to be an optic nerve sheath meningioma. Small perineural cysts have been reported with optic nerve sheath meningioma but we believe this to be the first reported case of a large peritumoral cyst associated with this type of mass lesion. We speculate on the aetiology of the cyst and the associated acute proptosis.
\end{abstract}

\section{CASE REPORT}

A previously fit and well 37-year-old woman presented to her general practitioner with left-sided proptosis of acute onset following a severe bout of coughing. This was associated with pain behind the eye and blurring of vision. There was no previous history of visual problems. Clinical examination revealed reduced visual acuity on the left to $6 / 36$, corrected to $6 / 12$. Right eye acuity was normal at $6 / 5$. There was a left-sided $5 \mathrm{~mm}$ non-axial, non-pulsatile proptosis, associated with limitation of eye movement in all extremes of gaze. Fundoscopy revealed a hyperaemic optic nerve head and blurring of the nasal disc margin. Choroidal folds were seen in the macular region. Systemic examination was normal.

Magnetic resonance imaging (MRI) of the orbits was performed at $0.5 \mathrm{~T}$ (GE Vectra). This revealed a partly solid, partly cystic, mass lesion behind the globe surrounding the optic nerve (Fig. 1a,b). The cystic area was seen superior to the solid component and appeared separate from the optic nerve (Fig. 2).

From: Departments of ${ }^{1}$ Neuroradiology and ${ }^{2}$ Ophthalmology, Manchester Royal Infirmary; Departments of ${ }^{3}$ Pathological Sciences and ${ }^{4}$ Diagnostic Radiology, Manchester University Medical School, Manchester, UK.

Correspondence to: $\mathrm{Dr}$ R. D. Laitt, Department of Neuroradiology, Manchester Royal Infirmary, Oxford Road, Manchester M13 9WL, UK. Fax: +44 (161)-272 8085.
There was no evidence of haemorrhage within the mass. Following contrast administration the solid part of the lesion demonstrated homogeneous enhancement (Fig. 3).

The tumour was resected via a left fronto-orbital approach which allowed exposure of the optic nerve following opening of the optic canal. The large cystic component of the mass demonstrated a good plane of cleavage from the surrounding orbital tissues. The deeper solid components appeared to be arising from the optic nerve in its immediate retro-globar portion. During dissection the cystic part of the mass ruptured, releasing a yellow serous fluid. The optic nerve was coagulated and divided $5 \mathrm{~mm}$ from its entry into the optic canal. The nerve was then reflected forwards from this point. The proximal part of the optic nerve within the orbit was then divided flush with the posterior aspect of the globe, allowing removal of the optic nerve and the associated tumour. The patient made an uneventful recovery following surgery.

Histological examination of the optic nerve and tumour demonstrated tumour cells in a syncytial arrangement with open nuclei, some showing cytoplasmic invagination (Fig. 4a) extending through the dura and into adjacent tissues. Immunohistochemistry demonstrated positivity for vimentin (Fig. 4b) and epithelial membrane antigen (EMA). The lesion was negative for glial fibrillary acidic protein (GFAP), S-100, neurone-specific enolase (NSE) and smooth muscle actin. These features are entirely consistent with a diagnosis of meningothelial meningioma. Clear cells were seen in the region of the cyst wall. No tumour cells were seen in the wall itself.

\section{DISCUSSION}

Meningiomas are the commonest optic nerve sheath tumours in adulthood. They usually present with gradually increasing proptosis and visual loss. Classically there is disproportion between the degree 


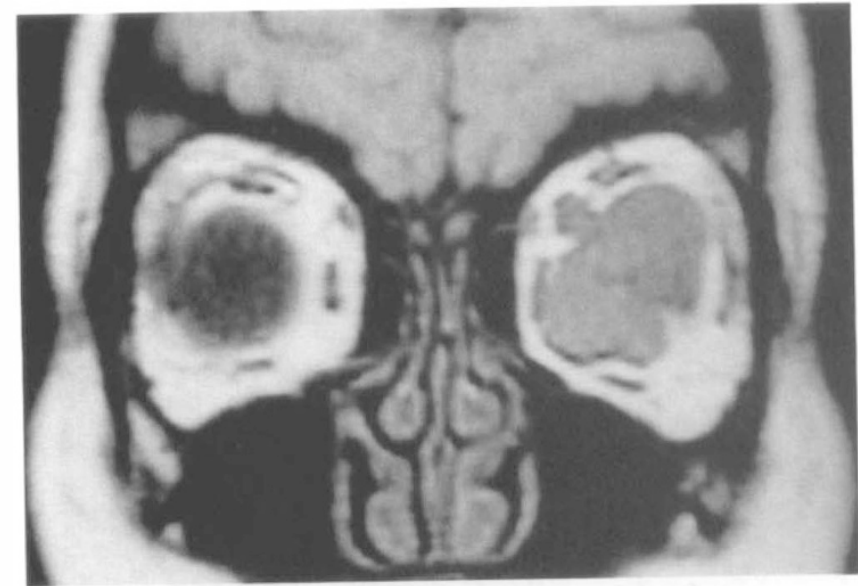

(a)

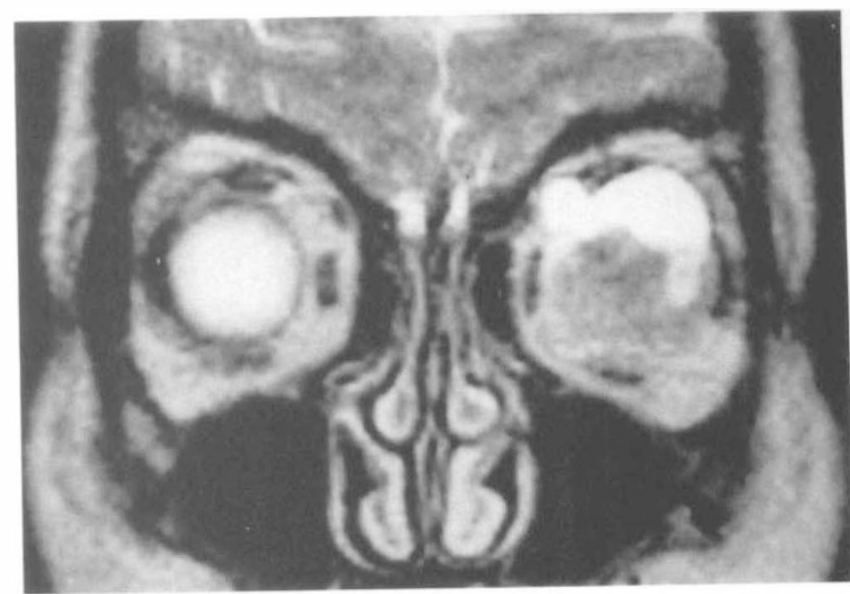

(b)

Fig. 1 (a) A T1-weighted coronal MRI scan demonstrating the retro-orbital mass with an area of low signal superiorly. (b) A T2-weighted coronal MRI scan demonstrates high signal in this region, consistent with a cyst capping the solid part of the lesion.

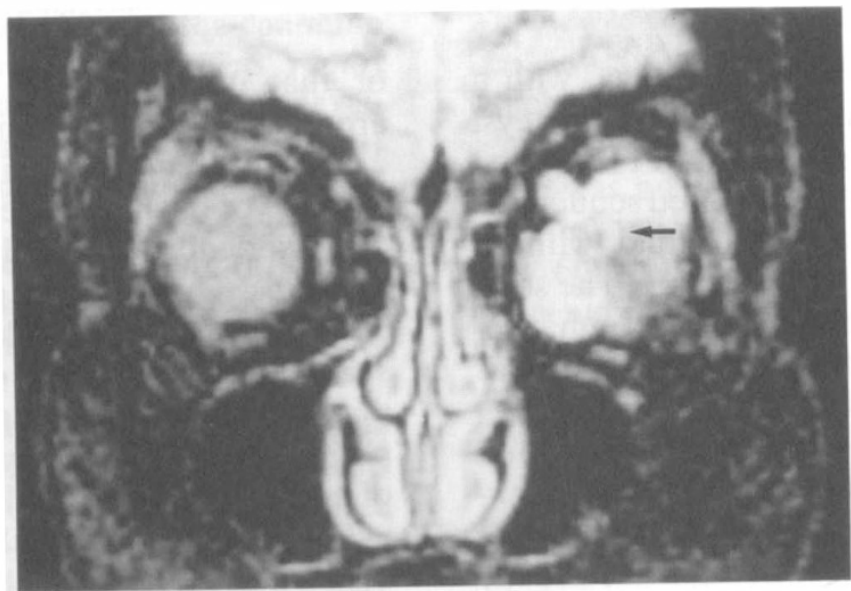

Fig. 2. A short tau inversion recovery (STIR) $M R I$ sequence suppresses the signal from retro-orbital fat and demonstrates the cerebrospinal fluid sheath around the optic nerve (arrow) to be separate from the cyst.

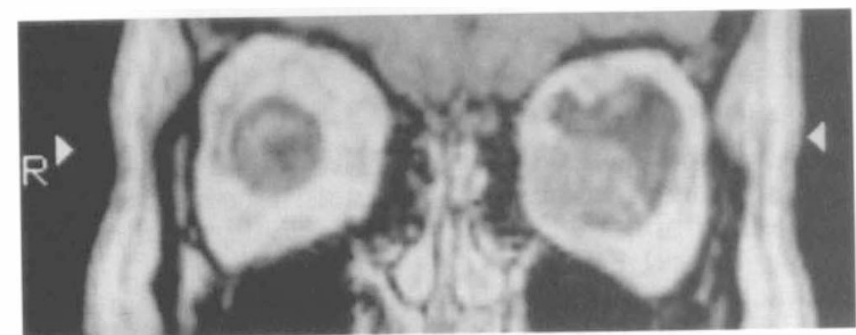

(a)

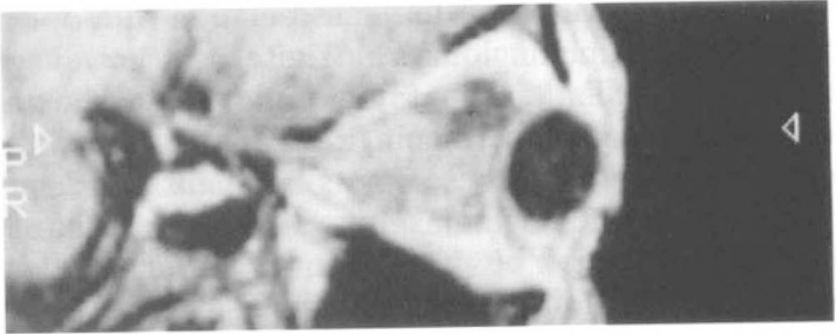

(b)

Fig. 3. (a),(b) T1-weighted coronal and oblique parasagittal MRI images after intravenous contrast (gadopentatate dimeglumine) demonstrate enhancement of the solid part of the tumour, which is intimately related to the optic nerve.

of visual loss and the degree of proptosis. ${ }^{1}$ Diplopia can also occur if the tumour compromises extraocular muscle function or produces significant choroidal folds. Clinical examination may reveal an enlarged blind spot or a centro-cecal scotoma.

Non-traumatic acute proptosis is an uncommon clinical presentation for optic nerve sheath meningioma. It is usually secondary to an underlying vascular lesion such as carotico-cavernous fistula or to haemorrhage into a mass lesion such as a lymphangioma or vascular malformation. To our knowledge there are no reports in the literature of optic nerve sheath meningioma presenting in this way.

Imaging in this case revealed some unusual features. There was a solid retro-orbital'mass lesion intimately related to the optic nerve producing proptosis of the globe. This had an atypical 


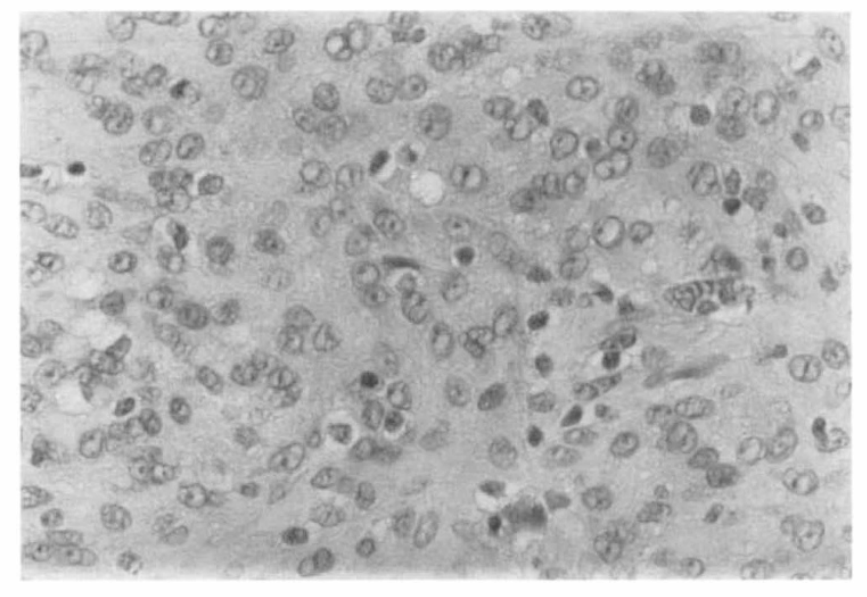

(a)

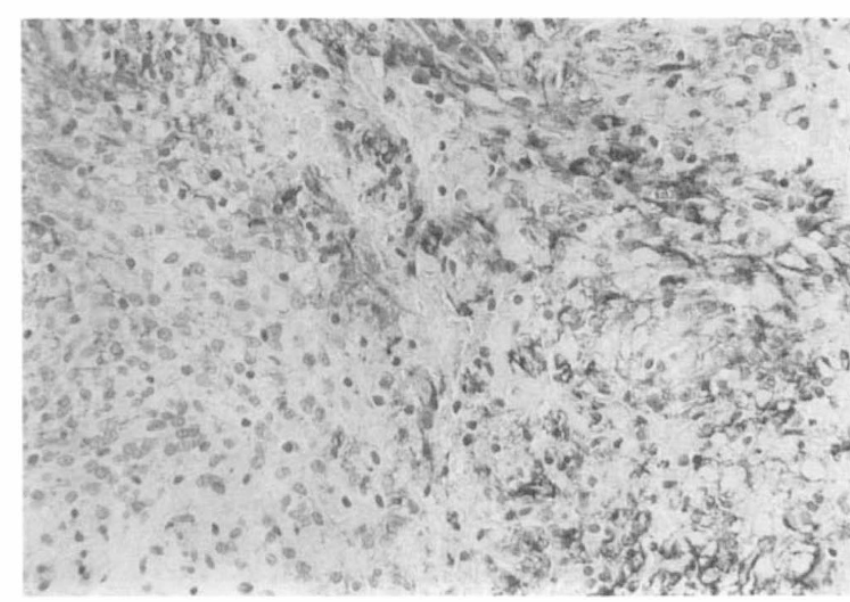

(b)

Fig. 4. (a) Tumour cells in a syncytial arrangement with open nuclei, some of which show cytoplasmic invagination. There is no mitotic activity or necrosis (haematoxylin \& eosin, $\times 295$ ). (b) Immunohistochemistry with anti-vimentin antibodies demonstrates spindle-shaped cells to be strongly positive (DAB-haematoxylin counterstain, $\times 148)$.

appearance for an optic nerve meningioma, with a cyst capping the lesion. The contents of the cyst returned uniform low signal on T1-weighted sequences and high signal on T2-weighted sequences. These are the signal characteristics of fluid, with no evidence to suggest previous haemorrhage. The solid part of the tumour demonstrated diffuse enhancement following contrast administration. There was no evidence of invasion of extraocular muscles.

The differential diagnosis of a partly solid and partly cystic retro-orbital lesion is limited. Lymphangiomas are characteristically poorly defined and multilobulated with cystic elements, but these usually contain haemorrhage in various stages of evolution. ${ }^{2}$ The lesion in this case had some of these features but the solitary cyst, absence of haemorrhage and welldefined tumour margin were against this diagnosis.

Cysts have been reported in association with optic nerve sheath meningiomas. ${ }^{3,4}$ These are, however, small cystic dilatations of the optic nerve sheath occurring distal to the meningioma. The mechanism of production of these cysts remains unclear. Intracranial meningiomas may have associated cysts, which may be intratumoral but are more commonly peritumoral. ${ }^{5}$ These cysts may be lined by tumour cells or by reactive gliotic tissue or may be an extension of the surrounding subarachnoid space. The cystic area seen on MRI in our case did not appear to conform to the cysts previously reported in association with nerve sheath meningioma as it lay superior to the mass and was much larger in size. It is possible that it represented a true peritumoral cyst as previously described with intracranial meningiomas or that it was related to the subarachnoid space around the nerve sheath.

The presentation with acute proptosis in the absence of haemorrhage suggests that the fluid-filled cyst suddenly increased in size, pushing the globe forwards. That this was apparently related to a bout of coughing, which is known to increase cerebrospinal fluid pressures, is of interest. It is possible that this produced either spontaneous rupture of the perineural sheath with cyst formation or enlargement of a pre-existing cystic dilatation of the sheath.

In conclusion we would like to emphasise the fact that optic nerve sheath meningioma can have a significant cystic component and can present with acute proptosis.

Key words: Optic nerve, Meningioma, Cyst, Magnetic resonance imaging.

\section{REFERENCES}

1. Jakobiec FA, Depot MJ, Kennerdell JS, et al. Combined clinical and computed tomographic diagnosis of orbital glioma and meningioma. Ophthalmology 1984;91:137-55.

2. Graeb DA, Rootman J, Robertson WD, Lapointe JS, Nugent RA, Hay EJ. Orbital lymphangiomas: clinical, radiological and pathological characteristics. Radiology 1990;175:417-21.

3. Lindblom B, Norman D, Hoyt WF. Perioptic cyst distal to optic nerve meningioma: MR demonstration. Am J Neuroradiol 1992;13:1622-4.

4. McNab AA, Wright JE. Cysts of optic nerve: three cases associated with meningioma. Eye 1989;3:355-9.

5. Pinna G, Beltramello A, Buffatti P, et al. Cystic meningiomas: an update. Surg Neurol 1986;26:441-52. 